\title{
Pulsation Powered by Deuterium Burning in Brown Dwarfs and Very-Low-Mass Stars
}

\author{
Ann Marie Cody \\ California Institute of Technology, MC 249-17, Pasadena, CA 91125
}

\begin{abstract}
Pulsation powered by deuterium burning in brown dwarfs and very low mass stars has been put forth (Palla \& Baraffe 2005) as a novel probe of the interiors of these objects in the 1-15 Myr age range. Previous observations have hinted at variability on the expected timescales of a few hours, suggesting but not confirming that the phenomenon is at work in young brown dwarfs. We have recently carried out a dedicated campaign to search for this putative class of pulsators among known low-mass members of five young star clusters. Our survey achieves sensitivity to periodic oscillations with photometric amplitudes down to several millimagnitudes. We present the census of variability over timescales ranging from minutes to days and discuss the current prospects for pulsation as a tool in the study of young, objects near the substellar boundary. As a byproduct, this work provides new insights into the distribution of stellar rotation periods at young ages via the detection of variability due to cool surface spots.
\end{abstract}

Keywords: brown dwarfs, low-mass stars, star clusters, stellar pulsations, stellar photometry, light curves PACS: $97.10 . \mathrm{Sj}, 97.20 . \mathrm{Vs}, 95.75 . \mathrm{Wx}$

\section{PULSATION AS A WINDOW INTO YOUNG BROWN DWARFS}

With masses between approximately 0.013 and $0.08 \mathrm{M}_{\odot}$ (13.6-83.8 $\mathrm{M}_{\text {Jup }}$ ), brown dwarfs (BDs) provide a crucial link between stellar and planetary evolution. Theoretical models $[1,2]$ are moderately successful in reproducing the observed properties of BDs, at least at intermediate and old ages [3]. Such models can be used to estimate fundamental parameters, namely mass and age. However the models have not been extensively tested by independent measurements of these fundamental quantities, nor do they account in detail for more complex realities such as magnetic fields and rotation which can have significant effects as illustrated for very low mass stars by [4]. Over the past dozen years, substantial populations of brown dwarfs have been identified at relatively young ages [5] and in star forming regions (e.g., [6]; [7]; [8]). They are observed to host intriguing phenomena such as disk accretion and mass outflow, similar to their more massive brethren, in addition to magnetic activity and atmospheric weather, common at all ages among BDs. What is needed now is correlation of theoretical predictions with observational phenomena in order to better constrain - using fundamental physics - the basic parameters of brown dwarfs. We discuss here one such possible new method.

Brown dwarfs are not typical targets for stellar seismology. However, the wealth of recently discovered young cluster populations as well as new theoretical modeling efforts offer incentive to search for pulsations in these objects. Palla \& Baraffe ([9]; hereafter PB05) have proposed that young $(\sim 1-15 \mathrm{Myr})$ BDs and very low mass stars (VLMSs) are subject to radial instability as a result of central deuterium burning and its strong temperature dependence (e.g., the $\varepsilon$ mechanism). Based on non-adiabatic models, they predict significant mode growth in a mass range from the BDs $\left(\mathrm{M} \sim 0.02 \mathrm{M}_{\odot}\right)$ to the VLMSs $\left(\mathrm{M} \sim 0.1 \mathrm{M}_{\odot}\right)$. The corresponding pulsation periods are expected to lie between one and five hours. Using a different set of models from [1] and a linear pulsation code, we have independently verified these basic results. Even more encouraging is the substantial overlap between available observational data for young BDs and VLMSs and the pulsation instability strip proposed by $\mathrm{PB} 05$, presented in Fig. 1 .

Although theoretical predictions provided the initial motivation to search for pulsation in young, low-mass cluster members, some observational evidence of periodic variability has been reported on the appropriate $\sim$ hour timescales. Several particularly promising pulsation candidates have been uncovered in the $\sigma$ Orionis cluster [10], [11], and [12]. Such variability is unlikely to be the result of rotational modulation of surface spots, since the short periods are inconsistent with rotational velocities previously derived for a handful of these objects [13].

We present here a dedicated observational campaign to photometrically monitor previously identified lowmass cluster members. Our observations are aimed at confirming the phenomenon of pulsation in young BDs, and could lead to the first anchoring of the low-mass end of interior models - upon which all studies of the initial mass function and star formation history are based. 


\section{OBSERVATIONAL CAMPAIGN}

\section{Search Space}

We have set out to perform a comprehensive photometric survey of BDs and VLMSs in a number of 1$5 \mathrm{Myr}$ star clusters. At these ages, objects below $0.1 M_{\odot}$ experience the deuterium burning stage of the pre-main sequence, making them potential pulsation candidates. We assembled a target sample from a set of confirmed and likely members of the IC 348 cluster, Upper Scorpius, Taurus, Chamaeleon I, and the $\sigma$ Orionis cluster. Spectral types are available for many objects, and we have employed the calibration of [14] to convert them to the temperature scale underpinning the brown dwarf models in PB05 [2]. Thus with a combination of photometric and spectroscopic data, we are able to place most known very low-mass young cluster members on the $\mathrm{H}$ $\mathrm{R}$ diagram. The result, as shown in Fig. 1, indicates a promising number of BDs and VLMSs on or near the instability strip. Because of measurement errors and possible model-dependent systematic effects in the position of the strip, we have sought to survey a large swath of parameter space above the zero-age main sequence.

Not only must our survey target objects favorable for pulsation, but its design must also take into account expected variability properties and limits. The linear theory of pulsation in BDs and VLMSs predicts periods of 1-4 hours, but cannot determine photometric variability amplitudes or the effect of convection on pulsation. We therefore designed observations to probe for pulsation to several millimagnitudes on timescales of a few hours. Since sensitivity to periodic signals depends on both signal-to-noise $(\mathrm{S} / \mathrm{N})$ and total number of data points, the detection limits reached with a 1-meter class telescope observing over one or two weeks are roughly equivalent to those achieved with a few nights with a larger telescope. We have selected the former set-up, since the longer time baseline offers increased frequency resolution and lower probability of weather interruptions.

The majority of our observations were carried out with the CTIO 1.0-meter telescope and the robotic Palomar 60 -inch telescope. For each selected cluster field, we observe for a total duration of 10-20 nights with continuous monitoring at 5-15 minute cadence (apart from occasional weather interruptions). We have aimed for $1 \%$ or better photometric precision, enabling identification of periodic variability in BDs with amplitudes as low as 0.005 magnitudes. Data are primarily in the $I$ band, where late-type objects are relatively bright but light is not dominated by potential dust disks.

These observations provide an unprecedented combination of cadence, precision, and comprehensive coverage of very low mass cluster members. As such, we have

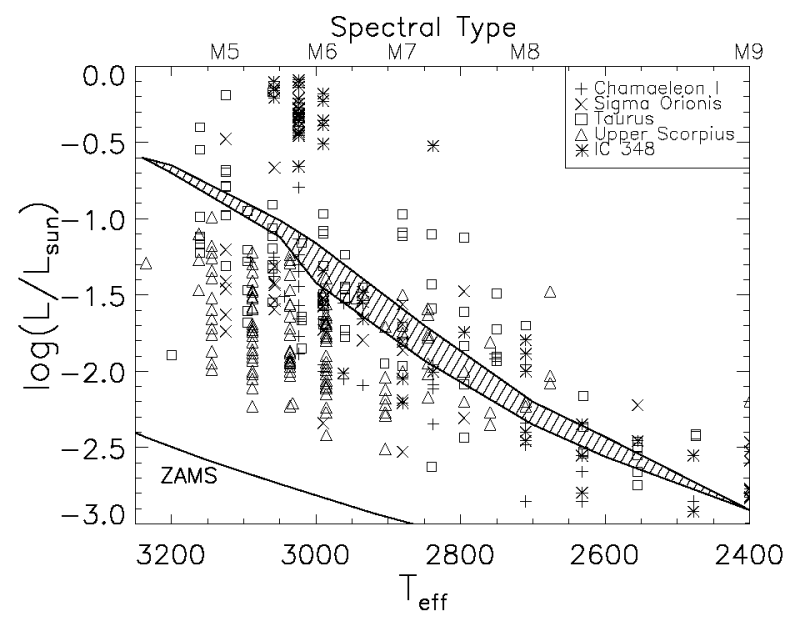

FIGURE 1. Many young cluster members are now known to fall on or near the deuterium-burning pulsation instability strip from PB05 (hashed area). Plotted here are effective temperatures and luminosities (in units of $L_{\odot}$ ) for BDs and VLMSs in the five clusters included in this photometric survey. As expected from the $\sim 1-5 \mathrm{Myr}$ age estimates, all points are well above the zero age main sequence (ZAMS). Data were drawn primarily from [14], [15], [16], [17], [18], and [19] and have typical errors of $100 \mathrm{~K}$ in temperature, and $0.15 \mathrm{dex}$ in $\log$ luminosity.

set out to confirm or place limits on the existence of pulsation by covering a statistically significant sample of deuterium-burning objects across different masses and ages. The campaign is nearing completion, with observations thus far of most BDs and VLMSs in IC 348, $\sigma$ Orionis, and Upper Scorpius, as well as about half of the intended survey regions in Cha I. Table 1 lists details on the five young clusters we have targeted, as well as observational time acquired; numbers of BDs and VLMSs listed refer to the entire cluster, and we list the fraction of these monitored.

\section{Variability Detection}

We have produced lightcurves for data obtained thus far with a combination of aperture and image-subtraction photometry. In non-crowded fields, we employ a modified version of the variable-aperture photometry program Vaphot [20]. Initial aperture sizes are chosen on an object-by-object basis so as to maximize the $\mathrm{S} / \mathrm{N}$ ratio. Aperture sizes are then scaled with the seeing full-widthhalf-maximum (FWHM) in subsequent images. This approach conserves the fraction of stellar flux measured while maintaining optimal S/N. For objects with close 
TABLE 1. Survey Coverage

\begin{tabular}{|c|c|c|c|c|c|}
\hline $\begin{array}{l}\text { Cluster } \\
\text { Region }\end{array}$ & $\begin{array}{c}\text { Approx. } \\
\text { Age }\end{array}$ & $\begin{array}{c}\text { Known BDs } \\
\text { \& VLMSs }\end{array}$ & Telescopes & $\begin{array}{l}\text { Nights } \\
\text { Observed }\end{array}$ & $\begin{array}{l}\text { Known BDs } \\
\text { covered }\end{array}$ \\
\hline IC 348 & 2-3 Myr & 32 & Palomar 60" & 10 & $75 \%$ \\
\hline$\sigma$ Orionis & 3-5 Myr & 29 & $\begin{array}{c}\text { CTIO 1m, } \\
\text { Palomar 200" }\end{array}$ & 27 & $75 \%$ \\
\hline Cha I & 1-3 Myr & 11 & CTIO $1 \mathrm{~m}$ & 13 & $25 \%$ \\
\hline $\begin{array}{l}\text { Upper } \\
\text { Scorpius }\end{array}$ & $5 \mathrm{Myr}$ & 5 & $\begin{array}{c}\text { Palomar } 60 " \\
\text { CTIO } 1 \mathrm{~m}\end{array}$ & 20 & $2 \%$ \\
\hline Taurus & 1-3 Myr & 2 & Palomar 60" & 12 & $2 \%$ \\
\hline
\end{tabular}

companions whose light contaminates the aperture or sky background, we have instead relied on the Hotpants image subtraction package [21] to remove flux from all nonvariable objects. We then perform aperture photometry on the output residual images. These procedures have resulted in photometric performance very close to the limits imposed by poisson noise and sky background. The typical lightcurve RMS as a function of $I$-band magnitude is presented in Fig. 2. The resulting light curves display several different types of variable behavior which must be investigated for signs of pulsation.

According to the theory of $\mathrm{PB} 05$, pulsation in BDs and VLMSs is expected to induce brightness fluctuations at a single frequency, which in theory can be identified with a power spectrum. However, it is not known a priori how many of the objects in our sample are host to multiple types of variability, some of which may be highamplitude and non-periodic. The plot of object magnitude versus lightcurve RMS in Fig. 2 offers a quick visual assessment of the global variability properties of our BDs and VLMSs. Objects whose lightcurves are inconsistent with a constant source appear as outliers, and we can statistically select them using a $\chi^{2}$ test. We model the median measurement uncertainty as a function of magnitude with the estimated poisson noise, sky background, and a small systematic error component. For variability detection at $>99 \%$ certainty, lightcurves must have a $\chi^{2}$ value greater than 6.6. Equivalently, we identify all data points lying more than a factor of 2.58 above the median photometric uncertainty as very likely variables.

To probe lightcurves for lower-level variability and search specifically for periodic signals, we employed the program Period04 [22], which computes an oversampled discrete fourier transform and may also be applied to time series with gaps. Periodic lightcurve variations appear as peaks in this periodogram. Since variability is concentrated in a narrow range of frequency space, we are able to probe to amplitudes at or below the level of the photometric precision, particularly at periods of $<5$ hours where noise is mostly uncorrelated. Based on [23],

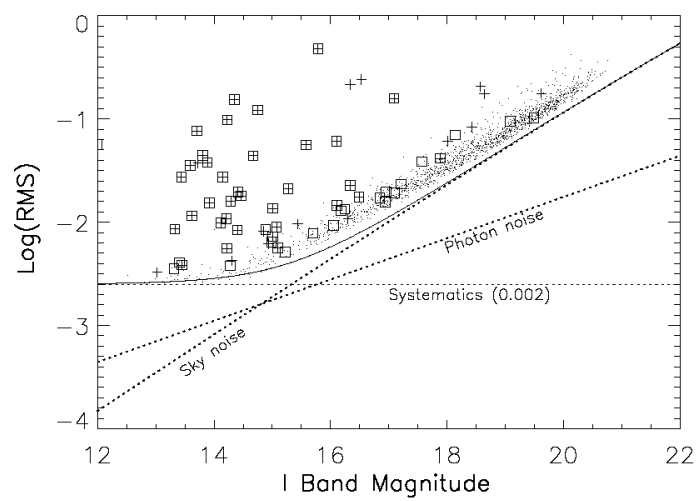

FIGURE 2. Example of photometric performance as a function of magnitude, from our data on the $\sigma$ Orionis cluster. Dots represent photometry of the entire field, while boxes denote likely cluster members and crosses mark objects that have passed one or more of our variability detection criteria.

we have selected a criterion of $\mathrm{S} / \mathrm{N}>4$ in the periodogram (where signal is the amplitude of variability) for detection of periodic variables at $99 \%$ confidence. For typical BDs with $I \sim 17$ and an expected pulsation period of $1-4$ hours, we are sensitive to variability amplitudes down to several millimagnitudes, as seen from the periodogram noise level in Fig. 4.

\section{RESULTS}

\section{Rotation and Accretion-related Variability}

While this study was initially designed to search for the putative new effect of pulsation in deuterium-burning objects, the large dataset has also uncovered numerous other phenomena, such as brightness modulation by rotating stellar spots, accretion-related fluctuations, and eclipses by companions. These effects are present in ob- 
jects across a wider range in mass than is expected for pulsation. Thus we can analyze and classify variable behavior in slightly higher mass stars $\left(M \sim 0.1-0.5 M_{\odot}\right)$, and use the results to help interpret lightcurves obtained for the subsample of BDs and VLMSs.

We find that fully $50 \%$ of young low-mass cluster members are variable within the M0-M9 spectral type range of our survey (e.g., $M \sim 0.02-0.5 M_{\odot}$ ), as evident in Fig. 2. Approximately one third of these exhibit variations at the $\sim 10 \%$ level but lack detectable periodicities. As shown in the example at the top of Fig. 3, such aperiodic objects are likely accretors. In contrast, most of the remaining variables are unambiguously periodic with amplitudes at the few percent level and likely indicative of rotating spots, as seen at the bottom of Fig. 3 . Their period distribution peaks near 1.8 days, with a tail toward the observing duration of $10-15$ days. The average amplitude of periodic variables is 0.03 magnitudes, although this value may be biased by our sensitivity limits.

\section{Signs of Pulsation in Brown Dwarfs?}

Periodic variability at the few percent level is certainly common amongst low-mass pre main sequence cluster members. But can any of it be attributed to pulsation instead of spots? In the absence of multi-band data, timescale is the main factor available to distinguish the two phenomena. While rotation periods less than one day are not unheard of for young very low mass stars, variability on 1-2-hour timescales is exceedingly short to be explained by the passage of rotating spots. Typical rotation periods seen amongst young cluster objects seem to to be around 1-10 days [24], although there is some evidence that this decreases into the brown dwarf regime [25]. In addition, rotational velocities corresponding to a 1-hour period would reach $100 \mathrm{~km} \mathrm{~s}^{-1}$ and higher, uncomfortably close to the break-up speeds estimated for objects at these ages. Consequently, we consider any objects with variability periods under $\sim 5$ hours to be prime pulsation candidates.

Despite sensitivity to millimagnitude amplitudes, the results of our periodogram analysis indicate a dearth of variables with periods less than 1 day. Furthermore, most of the brown dwarf targets show a surprising lack of variability on any timescale. Contrary to previous reports ([10]; [11]; [12]), we do not observe definitive variability with periods shorter than 15 hours. This is somewhat unexpected given the copious spot-related variability observed in slightly higher mass objects, and may be indicative of physical trends in the surface properties of brown dwarfs.

Interpretation of these results depends crucially on
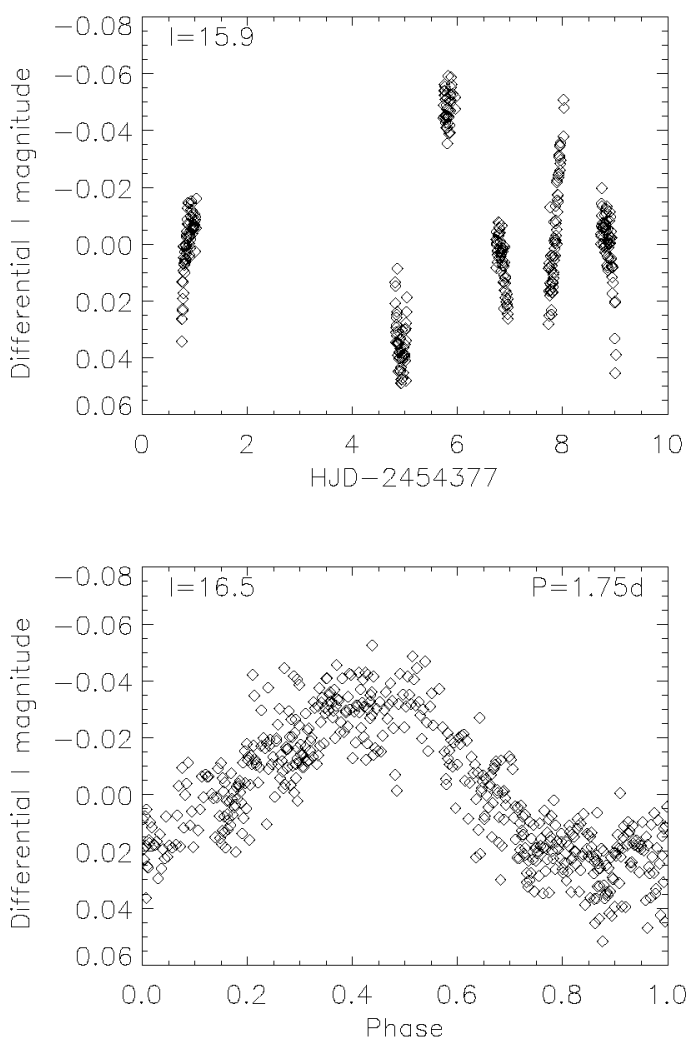

FIGURE 3. Lightcurves for two very-low-mass stars in IC 348 and $\sigma$ Orionis. Top: High-amplitude variability in this VLMS was confirmed with the $\chi^{2}$ test, whereas power spectrum analysis did not reveal any periodic frequencies. The stochastic nature of the variability thus suggests that the object is actively accreting. Bottom: A typical periodic variable, with 0.03 -magnitude amplitude. The lightcurve fluctuates with a period of 42 hours, a timescale suggestive of rotating spot features.

how many objects in the sample are actually on the narrow instability strip. Because of field-of-view constraints, we have not had the luxury of specifically targeting many of the best pulsation candidates. But observational uncertainties (typically $\sim 0.15$ dex in log luminosity and $100 \mathrm{~K}$ in temperature), possible intracluster age spreads, and the uncertain location of the strip itself also create difficulty in determining which objects are the best pulsation targets. Assuming that the width of the instability strip is approximately correct as calculated by PB05, we estimate that less than $30 \%$ of the BDs and VLMSs in our photometric sample falls on or near it. If we count only objects that are directly on the strip, this fraction drops to $22 \%$. Out of $\sim 50$ BDs and VLMSs in the sample, we therefore expect $\sim 10-15$ pulsators.

In reality, we have identified just a handful of cases thus far in which possible periodicities appear in the pe- 

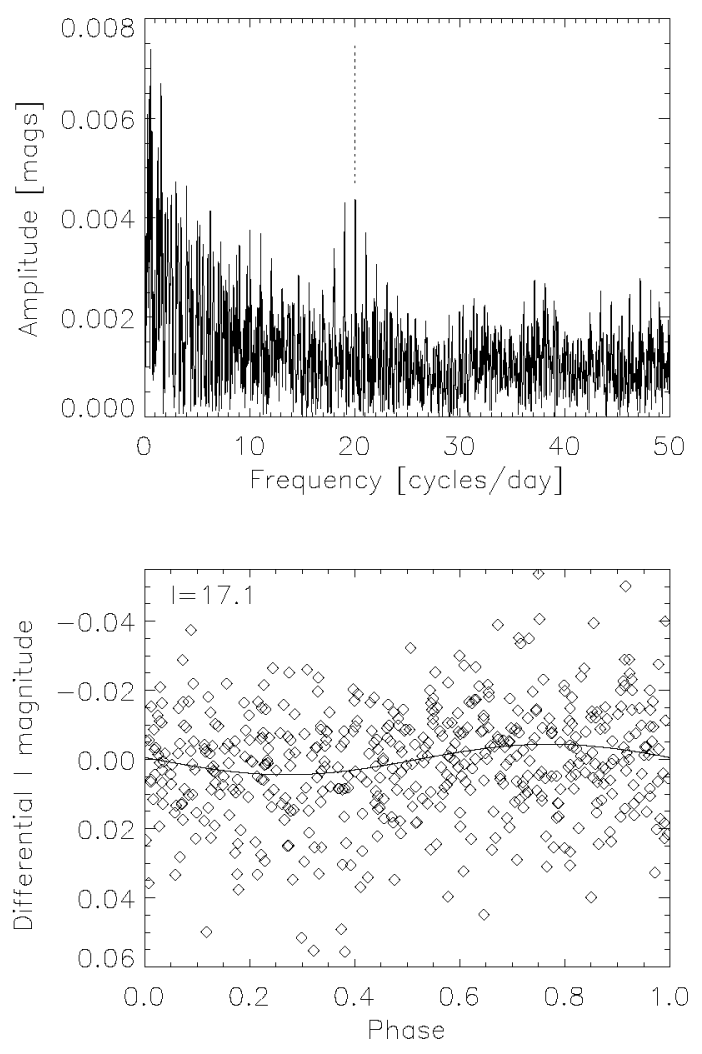

FIGURE 4. Top: The periodogram for a young brown dwarf in our sample shows a potential periodicity (marked with the dashed line) with amplitude 0.0044 magnitudes at $20.1 \mathrm{cy}-$ cles/day (1.2 hours). At low frequencies the data suffer from correlated ("red") noise, but beyond 10 cycles day ${ }^{-1}$ the noise level in the periodogram reaches 0.001 magnitudes. Hence this tentative detection appears at signal-to-noise of just over 4.0. Bottom: The light curve is phased to the possible signal. Due to the marginal nature of the detection, the signal cannot be confirmed by eye.

riodogram at 1-5-hour timescales. All of these are just above the detection limit at $\mathrm{S} / \mathrm{N} \gtrsim 4.0$; an example is presented in Fig. 4. Pulsation may be present in these 4-5 objects, but it is at such a low level that confirmation with a different instrument set-up is required. Future observational plans include follow-up observations of some of these most promising targets with both the Spitzer Space Telescope and Hubble Space Telescope.

In conclusion, we have presented an extensive photometric monitoring campaign on 1-5 Myr BDs and VLMSs, in hopes of confirming whether some of these objects are host to a new class of pulsation. While current evidence is not definitive, upcoming space-based data may provide the final word on variability in young, verylow-mass objects.

\section{REFERENCES}

1. A. Burrows, M. Marley, W. B. Hubbard, J. I. Lunine, T. Guillot, D. Saumon, R. Freedman, D. Sudarsky, and C. Sharp, ApJ 491, 856 (1997).

2. I. Baraffe, G. Chabrier, T. S. Barman, F. Allard, and P. H. Hauschildt, $A \& A$ 402, 701 (2003).

3. A. Burrows, W. B. Hubbard, J. I. Lunine, and J. Liebert, Reviews of Modern Physics 73, 719 (2001).

4. F. D'Antona, P. Ventura, and I. Mazzitelli, ApJL 543, L77 (2000).

5. R. Rebolo, E. L. Martin, G. Basri, G. W. Marcy, and M. R. Zapatero-Osorio, ApJL 469, L53 (1996).

6. K. L. Luhman, J. Liebert, and G. H. Rieke, ApJL 489, L165 (1997).

7. J. R. Najita, G. P. Tiede, and J. S. Carr, ApJ 541, 977 (2000).

8. C. L. Slesnick, L. A. Hillenbrand, and J. M. Carpenter, ApJ 610, 1045 (2004).

9. F. Palla, and I. Baraffe, $A \& A$ 432, L57-L60 (2005), arXiv: astro-ph/0502042.

10. C. A. L. Bailer-Jones, and R. Mundt, $A \& A$ 348, 800 (1999).

11. M. R. Zapatero Osorio, J. A. Caballero, V. J. S. Béjar, and R. Rebolo, $A \& A$ 408, 663 (2003).

12. J. A. Caballero, V. J. S. Béjar, R. Rebolo, and M. R. Zapatero Osorio, $A \& A$ 424, 857 (2004).

13. S. Mohanty, R. Jayawardhana, and G. Basri, ApJ 626, 498 (2005).

14. K. L. Luhman, J. R. Stauffer, A. A. Muench, G. H. Rieke, E. A. Lada, J. Bouvier, and C. J. Lada, ApJ 593, 1093 (2003).

15. K. L. Luhman, ApJS 173, 104 (2007).

16. D. Barrado y Navascués, V. J. S. Béjar, R. Mundt, E. L. Martín, R. Rebolo, M. R. Zapatero Osorio, and C. A. L. Bailer-Jones, $A \& A$ 404, 171 (2003).

17. D. Ardila, E. Martín, and G. Basri, $A J$ 120, 479487 (2000).

18. K. L. Luhman, ApJ 645, 676-687 (2006).

19. C. L. Slesnick, J. M. Carpenter, L. A. Hillenbrand, and E. E. Mamajek, $A J$ 132, 2665-2674 (2006).

20. H. J. Deeg, and L. R. Doyle, "VAPHOT - A Package for Precision Differential Aperture Photometry," in Third Workshop on Photometry, p. 85, edited by W. J. Borucki, and L. E. Lasher, 2001, p. 85.

21. A. C. Becker, D. M. Wittman, P. C. Boeshaar, A. Clocchiatti, I. P. Dell'Antonio, D. A. Frail, J. Halpern, V. E. Margoniner, D. Norman, J. A. Tyson, and R. A. Schommer, ApJ 611, 418 (2004).

22. P. Lenz, and M. Breger, Communications in Asteroseismology 146, 53 (2005).

23. M. Breger, J. Stich, R. Garrido, B. Martin, S. Y. Jiang, Z. P. Li, D. P. Hube, W. Ostermann, M. Paparo, and M. Scheck, $A \& A$ 271, 482 (1993).

24. W. Herbst, J. Eislöffel, R. Mundt, and A. Scholz, "The Rotation of Young Low-Mass Stars and Brown Dwarfs," in Protostars and Planets $V$, edited by B. Reipurth, D. Jewitt, and K. Keil, 2007, pp. 297-311.

25. M. V. Rodriguez-Ledesma, R. Mundt, and J. Eislöffel, ArXiv e-prints (2009), 0906.2419. 